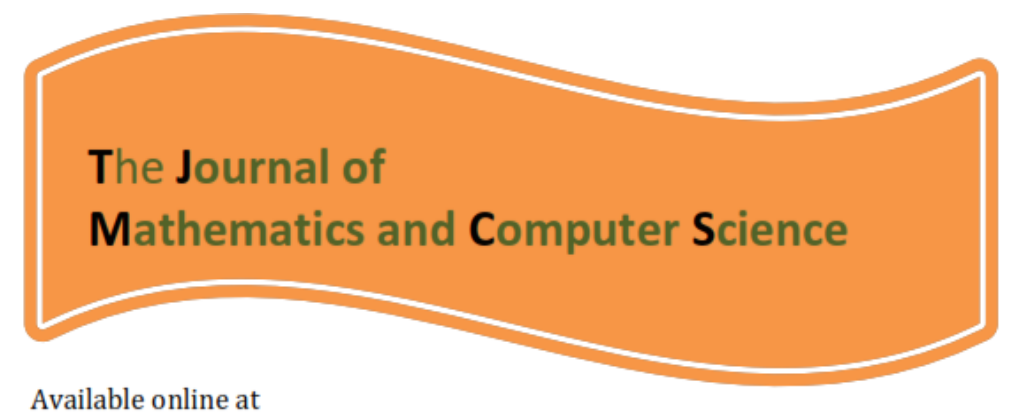

http://www.TJMCS.com

The Journal of Mathematics and Computer Science Vol. 4 No.1 (2012) 102 - 109

\title{
A Combined Method for the Numerical Solution of Boundary Value Problems of second order
}

\author{
R. Darzi ${ }^{1}$, A. Neamaty ${ }^{2}$, Y. Darzi, B Mohammadzadeh ${ }^{3}$ \\ ${ }^{1}$ Department of Mathematics, Neka Branch, Islamic Azad University, Neka, Iran \\ Darzi@iauneka.ac.ir \\ ${ }^{2}$ Department of Mathematics, University of Mazandaran, Babolsar, Iran \\ Neamaty@math.com \\ ${ }^{3}$ Department of Mathematics, Sari Branch, Islamic Azad University, Sari, Iran \\ Bahar@math.com
}

Received: December 2011, Revised: March 2012

Online Publication: May 2012

\begin{abstract}
In this work, a technique based on combination between collocation and spline method along with the shooting method is proposed for the solution of boundary value problem of order 2. Numerical results show that the method is simple and effective.
\end{abstract}

Keywords: Boundary value problem, Collocation method, Shooting method.

\section{Introduction}

It is not in general easy to find a closed-form solution of differential equations $[1,2]$. A part form numerical integration methods, several analytical ones, such as Adomian decomposition method [3-6] and the variational iteration method (VIM) [7-12] are proposed to find approximate, and if possible in closed form, solutions of differential equations. In this paper, we discuss the numerical solution of boundary value problem of the form

$$
\left[a_{2} D^{2}+a_{1} D+a_{0} D^{0}\right] y(t)=f(t), \quad 0<t<T,
$$

subject to

$$
y(0)=\alpha \quad y(T)=\beta .
$$


where $a_{0}, a_{1}, a_{2}, \alpha$ and $\beta$ are constants with $a_{2} \neq 0$ and $y \in L_{1}[0, T]$.

The method of solution is based on a conjugating collocation and spline analysis combined with multiple shooting method. Note that similar study can be done for a general case when the boundary conditions are

$$
\left\{\begin{array}{l}
M_{1} y(0)+N_{1} y^{\prime}(0)=\alpha, \\
M_{2} y(T)+N_{2} y^{\prime}(T)=\beta,
\end{array}\right.
$$

where $M_{1}, N_{1}, M_{2}, N_{2}, \alpha$ and $\beta$ are constants.

The use of spline approximation [13] and collocation method [14] has been effectively implemented for the solution of initial value problem. Additionally, the cubic spline collocation method to solve two classes of special boundary value problem has been used [15].

\section{Method of solution}

The following is a brief derivation of the algorithm used to solve problem (1) subject to (2).

\subsection{Collocation method}

For sake of simplicity we discuss the solution of (1) as initial value problem with

$$
y(0)=\alpha, \quad y^{\prime}(0)=\hat{\beta}_{1},
$$

where $\hat{\beta}_{1}$ is unknown constant which will be determined later. We firstly subdivide the interval $[0, T]$ into $N$ uniform subintervals $I_{n}=\left[t_{n}, t_{n+1}\right]$ where $n=0, \ldots, N-1$. Let $H_{N}=\left\{t_{n}=n h: n=0, \ldots, N-1\right\}$ with $h=\frac{T}{N}$. Assume that the exact solution of Eq.(1) subject to condition (3) can be approximated by an element $u$ in the space of piecewise polynomials of degree $m+d$ which are $d$-times continuously differentiable on $I$ represented by

$$
S_{m+d}^{(d)}\left(H_{N}\right)=\left\{u \in C^{(d)}(I):\left.u\right|_{\sigma_{n}} \text { is a polynomial of degre } m+d\right\}
$$

for known integers $m \geq 1$ and $d \geq 0$ (the number of the initial conditions minus one i.e. $d=1$ in the present work). It should be noted that the integer $m$ represents the number of collocation points in each subinterval $I_{n},(n=0, \ldots, N-1)$; those points are defined as

$$
X_{n}=\left\{t_{n, i}=t_{n}+c_{i} h: i=1, \ldots, m\right\}, \quad 0<c_{1} \leq \ldots \leq c_{m} \leq 1
$$


Consequently, the exact solution, $y$, of (1) and (3) needs to be approximated on $I$ by an element $u \in S_{m+d}^{(d)}\left(Z_{N}\right)$ satisfying

$$
\left[a_{2} D^{2}+a_{1} D+a_{0} D^{0}\right] u(t)=f(t), \quad t \in \bigcup_{n=0}^{N-1} X_{n},
$$

subject to

$$
u(0)=\alpha, \quad u^{\prime}(0)=\hat{\beta}_{1},
$$

On each subinterval, $I_{n}$, the spline $u$ can be presented as a piecewise polynomials of degree $m+d$ of the form

$$
u(t)=u_{n}\left(t_{n}+\tau h\right)=\sum_{s=0}^{d} a_{s}^{(n)} \tau^{s}+\sum_{r=1}^{m} b_{r}^{(n)} \tau^{d+r}, \quad t \in I_{n}
$$

where $\tau \in[0,1]$.

Substituting (6) into (4) one obtains

$$
\begin{gathered}
a_{2} h^{-2}\left[\sum_{s=2}^{d} s(s-1) a_{s}^{(n)} c_{i}^{s-2}+\sum_{r=1}^{m}(d+r)(d+r-1) b_{r}^{(n)} c_{i}^{d+r-2}\right] \\
+a_{1} h^{-1}\left[\sum_{s=1}^{d} s a_{s}^{(n)} c_{i}^{s-1}+\sum_{r=1}^{m}(d+r) b_{r}^{(n)} c_{i}^{d+r-1}\right]+a_{0}\left[\sum_{s=0}^{d} a_{s}^{(n)} c_{i}^{s}+\sum_{r=1}^{m} b_{r}^{(n)} c_{i}^{d+r}\right]=f\left(t_{n, i}\right),
\end{gathered}
$$

where $i=1, \ldots, m$. It can be shown that the above equations can be expressed in the following $m \times m$ matrix form

$$
\mathbf{V} \mathbf{b}^{(n)}=\mathbf{U} \mathbf{a}^{(n)}+\mathbf{F}
$$

where

$$
\begin{array}{llrl}
(\mathbf{V})_{i, r} & =a_{2}(d+r)(d+r-1) c_{i}^{d+r-2}+a_{1} h(d+r-1) c_{i}^{d+r}+a_{0} h^{2} c_{i}^{d+r} . & i, r=1, \ldots, m, \\
(\mathbf{U})_{i, s} & =a_{2} s(s-1) c_{i}^{s-2}+a_{1} h s c_{i}^{s-1}+a_{0} h^{2} c_{i}^{s}, & i=1, \ldots, m ; & s=0, \ldots, d, \\
(\mathbf{F})_{i, 1}=h^{2} f\left(t_{n, i}\right), & i=1, \ldots, m .
\end{array}
$$

In general, $\mathbf{a}^{(n)}=\left[a_{0}^{(n)}, \ldots, a_{d}^{(n)}\right]^{t}$ and $\mathbf{b}^{(n)}=\left[b_{1}^{(n)}, \ldots, b_{m}^{(n)}\right]^{t}$, where [. $]^{t}$ means the transpose of the vector. Note that when $n=0$, the vector $\mathbf{a}^{(0)}$ is known from the initial conditions, i.e. $\mathbf{a}^{(0)}=\left[\frac{h^{s}}{s !} \frac{d^{s} y}{d t^{s}}(0)\right]^{t} ; s=0, \ldots, d$. On the other hand, when $n \geq 1$, we have to use the smoothness conditions at $t=t_{n}$ which leads to a relation between the unknown vector 
$\mathbf{a}^{(n+1)}$ and the known vectors $\mathbf{a}^{(n)}$ and $\mathbf{b}^{(n)}$.

$$
\mathbf{H}=\left(\begin{array}{cccccccccc}
1 & 1 & 1 & 1 & 1 & 1 & . & . & . & 1 \\
0 & 1 & 2 & 3 & 4 & 5 & . & . & . & l-1 \\
0 & 0 & 1 & 3 & 6 & 10 & . & . & . & \prod_{i=1}^{2} \frac{(l-i)}{2 !} \\
0 & 0 & 0 & 1 & 4 & 10 & . & . & . & \prod_{i=1}^{3} \frac{(l-i)}{3 !} \\
0 & 0 & 0 & 0 & 1 & 5 & . & . & . & \prod_{i=1}^{4} \frac{(l-i)}{4 !} \\
0 & 0 & 0 & 0 & 0 & 1 & . & . & . & \prod_{i=1}^{5} \frac{(l-i)}{5 !} \\
. & . & . & . & . & . & . & . & . & . \\
. & . & . & . & . & . & . & . & . & . \\
. & . & . & . & . & . & . & . & . & . \\
0 & 0 & 0 & 0 & 0 & 0 & 0 & 0 & 0 & 1
\end{array}\right)
$$

and

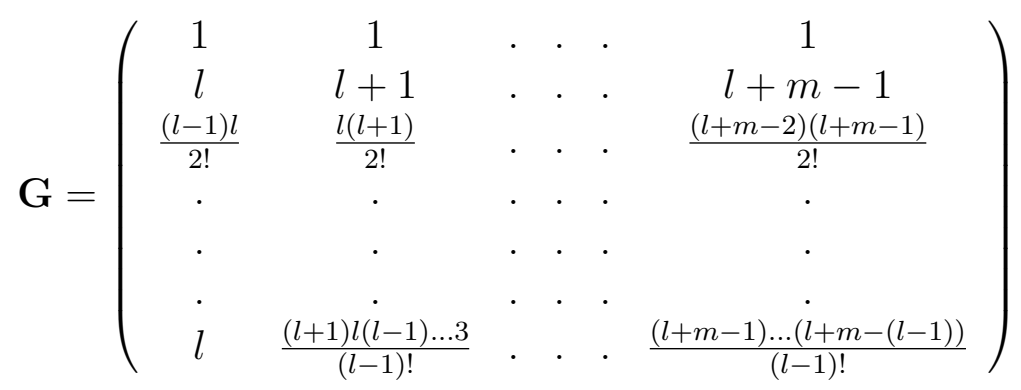

In the present work, this relation is given by $\mathbf{a}^{(n+1)}=\mathbf{H} \mathbf{a}^{(n)}+\mathbf{G} \mathbf{b}^{(n)}$ where $\mathbf{H}=\left(\begin{array}{ll}1 & 1 \\ 0 & 1\end{array}\right), \quad$ and $\quad \mathbf{G}=\left(\begin{array}{lll}1 & 1 & 1 \\ 2 & 3 & 4\end{array}\right)$

In the next subsection, we discuss the implementation of the multiple shooting method in the numerical solution of problem (1-2).

\subsection{Shooting method}

In the first, we rewrite problem (1-2) in the form

$$
L[y](t)=f(t), \quad t \in[0, T]
$$

subject to

$$
y(0)=\alpha, \quad y(T)=\beta,
$$

where

$$
\mathbf{L}=a_{2} D^{2}+a_{1} D+a_{0} D^{0}
$$

Firstly we partition the domain $[0, T]$ as

$0=T_{0}<T_{1}<\ldots<T_{L}=T$.

Hence, theoretically, the solution of problem (7-8) on the time interval $[0, T]$ can be determined by solving problem $(7-8)$ on the subintervals $\left[T_{l}, T_{l+1}\right],(l=0, \ldots, L-1)$. For 
the purpose of implementing shooting method, we added extra conditions at the grid points $T_{l},(l=0, \ldots, L-1)$, so that we have the following set of initial value problems

$$
L\left[u_{l}\right]=f(t), \quad t \in\left[T_{l}, T_{l+1}\right]
$$

subject to

$$
u_{l}\left(T_{l}\right)=\hat{\beta}_{2 l}, \quad u_{l}^{\prime}\left(T_{l}\right)=\hat{\beta}_{2 l+1}
$$

where $\hat{\beta}_{j},(j=1, \ldots, 2 L-1)$ are unknown real parameters and $\hat{\beta}_{0}=\alpha$. The solution of initial value problem (9-10) will be obtained by the method described in the previous subsection where the parameters $\hat{\beta}_{l},(l=0, \ldots, 2 L-1)$, will be determined by solving the following system of algebraic equations

$$
\begin{aligned}
& u_{0}\left(T_{1}, \hat{\beta}_{1}\right)=u_{1}\left(T_{1}, \hat{\beta}_{2}, \hat{\beta}_{3}\right), \\
& u_{0}^{\prime}\left(T_{1}, \hat{\beta}_{1}\right)=u_{1}^{\prime}\left(T_{1}, \hat{\beta}_{2}, \hat{\beta}_{3}\right), \\
& u_{1}\left(T_{2}, \hat{\beta}_{2}, \hat{\beta}_{3}\right)=u_{2}\left(T_{2}, \hat{\beta}_{4}, \hat{\beta}_{5}\right), \\
& u_{1}^{\prime}\left(T_{2}, \hat{\beta}_{2}, \hat{\beta}_{3}\right)=u_{2}^{\prime}\left(T_{2}, \hat{\beta}_{4}, \hat{\beta}_{5}\right), \\
& \vdots \\
& u_{k-2}\left(T_{k-1}, \hat{\beta}_{2 k-4}, \hat{\beta}_{2 k-3}\right)=u_{k-1}\left(T_{2}, \hat{\beta}_{2 k-2}, \hat{\beta}_{2 k-1}\right), \\
& u_{k-2}^{\prime}\left(T_{k-1}, \hat{\beta}_{2 k-4}, \hat{\beta}_{2 k-3}\right)=u_{k-1}^{\prime}\left(T_{2}, \hat{\beta}_{2 k-2}, \hat{\beta}_{2 k-1}\right), \\
& u_{k-1}\left(T, \hat{\beta}_{2 k-2}, \hat{\beta}_{2 k-1}\right)=\beta
\end{aligned}
$$

which can be solved numerically using several numerical techniques such as Newtons method. In summary, this technique is called multiple shooting technique of order $L$.

\section{$3 \quad$ Numerical results}

Example1. Consider the following differential equation

$$
y^{\prime \prime}(t)+\frac{1}{16} y(t)=0, \quad 0<t<2 \pi
$$

subject to

$$
y(0)=0, \quad y(2 \pi)=6.5244 \text {. }
$$

Applying the multiple shooting method of order four requires dividing the domain $[0,2 \pi]$ into four subinterval as follows $0=T_{0}<T_{1}=\pi<T_{2}=2 \pi$. 


$$
\begin{array}{ccc}
L\left[u_{0}\right]=0, \quad 0<t<1, \quad u(0,:)=0, & u^{\prime}(0,:)=\hat{\beta}_{1} \\
L\left[u_{1}\right]=0, \quad 1<t<2, \quad u(1,:)=\hat{\beta}_{2}, & u^{\prime}(1,:)=\hat{\beta}_{3},
\end{array}
$$

where

$$
L\left[u_{l}\right]=D^{2} u_{l}(t)+D u_{l}(t)+u_{l}(t), \quad l=0,1
$$

Herein

$u_{0}(t,:)=u_{0}\left(t, \hat{\beta}_{1}\right), u_{1}(t,:)=u_{l}\left(t, \hat{\beta}_{2}, \hat{\beta}_{3}\right)$.

Each initial value problem of (13-14) will be solved using the collocation method where we use $m=3$ with the collocation parameters

$$
\left(\begin{array}{l}
c_{1} \\
c_{2} \\
c_{3}
\end{array}\right)=\left(\begin{array}{c}
\frac{x_{1}+1}{2} \\
\frac{x_{2}+1}{2} \\
\frac{x_{3}+1}{2}
\end{array}\right), \quad \quad x_{i}=\cos \left(\frac{2 i+1}{6}\right), \quad i=0,1,2 .
$$

The $x_{i}, i=0,1,2$, are the roots of the Tchybechev polynomial of degree 3 . Note that, it is difficult to find a specific optimum choice for $m$.

Finally, the values for the parameters $\beta_{i}, i=1,2,3$ are determined by solving the following algebraic system

$u_{0}\left(\pi, \hat{\beta}_{1}\right)=u_{1}\left(\pi, \hat{\beta}_{2}, \hat{\beta}_{3}\right), \quad u_{0}^{\prime}\left(\pi, \hat{\beta}_{1}\right)=u_{1}^{\prime}\left(\pi, \hat{\beta}_{2}, \hat{\beta}_{3}\right)$

$u_{1}\left(2 \pi, \hat{\beta}_{2}, \hat{\beta}_{3}\right)=6.5422$.

Using matlab package we get

$\hat{\beta}_{1}=1, \quad \hat{\beta}_{2}=1.3782$,

$\hat{\beta}_{3}=1.7628$.

\section{Conclusions}

In his paper, we apply a conjugating collocation and spline analysis combined with shooting method for solving boundary value problems of order 2 subject to boundary conditions. It may be concluded that the method is powerful and efficient technique for finding approximate solutions for wide classes of differential equations. In the example, we have proposed the numerical result by using matlab package. 


\section{References}

[1] J.H. He, The multistage variational iteration method for a class of nonlinear system of ODEs, Physica Scripta, 76 (2007)388-392.

[2] J.H. He, Variational iteration method for autonomous ordinary differential system, Appl. Mech. Comput, 114 (2000) 115-123.

[3] J. Biazar, E. Babolian, R. Islam, Solution of ordinary differential equations by Adomian decomposition method, Appl. Mech. Comput, 147, (3) (2004) 713-719.

[4] J. Biazar, R. Islam, Solution of wave equation by Adomian decomposition method and thr restrictions of the method, Appl. Mech. Comput, 147, (3) (2004) 713-719.

[5] D. Kaya, M.E. El-Sayed, A numerical solution of thr Klein-Gordon equation and converegence of the decomposition method, Appl. Mech. Comput, 156, (2) (2004) 341-353.

[6] S. Momani, Z .Odibat, Analytical solution of a time-fractional-stokesequation by adomian decomposition method, Appel. Math. Comput, 177 (2) (2006) 488-494.

[7] A. Neamaty, R. Darzi, Variational iteration method for eigenvalues of fractional sturm-liouville problem, Aus. J. Basic. Appl. Sci, (4) (2010) 646-651.

[8] A. Neamaty, R. Darzi, Comparison between the Variational iteration method and the Homotopy perturbation method for the Sturm-Liouville differential equation, Boundary value problems, in press, doi:10.1155/2010.317369.

[9] D.D. Ganji, M. Nurollahi, M. Rostamian, a comparison of Variational iteration method with Adomian decomposition method in some highly nonlinear equations, Int. J. Sci. Techn, (2) (2007) 179-188.

[10] M.A. Noor, S.T. Mohyud-din, Variational iteration method for solving initial and boundary value problems of Bratu-type, Int. J. Appl. Appl. Math, (3) (2008) 89-99.

[11] B. Batiha, M.S.M. Noorani, I. Hashim, Application of variational iteration method to a general Riccati equation, Int. Math. Froum, (2) (2007) 2759-2770. 
R. Darzi, A. Neamaty, Y. Darzi, B Mohammadzadeh/ TJMCS Vol. 4 No. 1 (2012) 102 - 109

[12] S.T. Mohyud-din, M.A. Noor, Variational iteration method for discrete KDV equation, Bull. .Inst. Math, (5) (2010) 69-73.

[13] L. Blank, Numerical treatment of differential equations of fractional order, Numerical analysis report 287, Manchester center Numer. Comput. Math, (1996).

[14] EA. Rawashdeh, Numerical solution of semidifferential equation by collocation method, Appl. Math. Comput, 174 (2006) 869-876.

[15] M. Lia, S. Jimenezc, N. Niea, T. Tanaga, L. Vazqueze, Solving two-point boundary value problems of fractional differential equation by spline collocation methods,

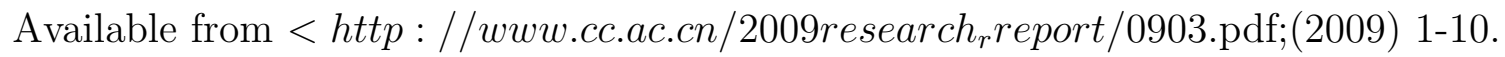

